\title{
INICIO DE LA DOCENCIA UROLÓGICA EN LA UNIVERSIDAD ESPAÑOLA. Dr. ALBERTO SUÁREZ DE MENDOZA
}

\author{
Mariano Pérez Albacete.
}

Servicio de Urología. Hospital Universitario Virgen de la Arrixaca. Murcia. España.

\begin{abstract}
Resumen.- OBJETIVO: Presentamos la semblanza del Dr. Suárez de Mendoza, uno de los primeros urólogos formado en Urología en el Hospital Necker, de París, junto al Dr. Guyon, que abrió el campo a la creación de la especialidad en España y el primer profesor oficial de la asignatura en la Facultad de Medicina de Madrid.

MÉTODO: Hemos revisado su expediente académico en el Archivo Histórico Nacional y buscado datos en la Facultad de Medicina y en el Colegio de Médicos madrileño sobre su presencia en España. Extraído de los Reales Decretos información sobre la escuela libre de Medicina y la creación de las especialidades médicas en los planes de estudios universitarios y, por último, analizamos su obra escrita.
\end{abstract}

RESULTADO/CONCLUSIÓN: Consideramos al Dr. Suárez de Mendoza una de las personalidades que dieron entidad a la Urología en nuestro país, por su formación, por su amplitud de saberes y por su vasta experiencia, como profesor de la asignatura en la Universidad española, como autor de un gran número de trabajos publicados y como inventor por sus aportaciones al desarrollo de la anestesia y de la Urología. Su tratado, el primero en español que recoge con detalle las primeras innovaciones en los medios de exploración urológicos, nos permite conocer y valorar el progresivo avance y evolución del conocimiento que presenta la Urología.

Palabras clave: Alberto Suárez Mendoza. Semblanza urológica. Docencia urológica en la Universidad. Historia de la Urología.

Summary.- OBJECTIVES: We present a biographical sketch of Dr. Suarez de Mendoza, one of the first urologists trained as so in the Necker Hospital in Paris with Dr. Guyon, who opened the field to the creation of this speciality in Spain, and first official professor of the subject of in the Faculty of Medicine of Madrid.

METHODS: We reviewed his academic expedient in the National Historical Archive and search for data about his presence in Spain in the Faculty of Medicine and Medical College of Madrid. From the Royal Decrees we obtained information about the Free School of Medicine, and the creation of medical specialities in the University study plans; finally, we analyzed his written works.

RESULTS /CONCLUSIONS: We consider Dr. Suarez de Mendoza one of the personalities giving entity to 
Urology in our country, for his education, for the amplitude of his knowledge, and for his wide experience, as professor of the subject in the Spanish University, as author of a great number of published works, and as inventor of his contributions to the development of anesthesia and urology. His treaty, the first in Spanish covering in detail the innovations in urological examination methods, enables us to know and value the progressive advance and evolution of knowledge in urology.

Keywords: Alberto Suárez Mendoza. Urological teaching. University. History of urology.

\section{INTRODUCCIÓN}

Alberto Raimundo Eugenio Suárez de Mendoza nació en Puerto Rico el 13 de noviembre de 1849, estudió Medicina en la Universidad de Madrid, a la que llegó con 15 años, y, tras licenciarse a los veinte, marcha a Francia donde ingresó en el ejército como cirujano y como tal participó en la campaña militar de 1870-71; se especializó en Urología en el Hospital Necker con el profesor Guyon del que se declara discípulo; en 1873 vio operar a Sir Spencer Wells, posiblemente en Londres, en 1875 realiza su doctorado en la Sorbona y también estuvo en Alemania para ampliar su formación, regresa a la ciudad de Sena a ejercer la profesión sin que encontremos más datos sobre su estancia en el país vecino.

En 1895, con cuarenta y cinco años de edad decide volver a España, el 7 de febrero de 1896, obtiene el título de doctor en la Universidad Central (Figura 1), tras la lectura de su memoria «La sinfiseotomía», "sección de la sífilis cartilaginosa de los huesos del pubis", (Figura 2) ante un tribunal presidido por D. Julián Calleja y compuesto por D. Francisco Criado Aguilar, José Grinda e lldefonso Rodríguez y en el que actuó de secretario el Dr. Ramón Jiménez.

En Madrid, imparte cursos de Urología en la escuela libre creada a instancia del Dr. Letamendi, la "Escuela Práctica de Especialidades Médicas" donde

\section{UNIVERSIDAD CENTRAL}

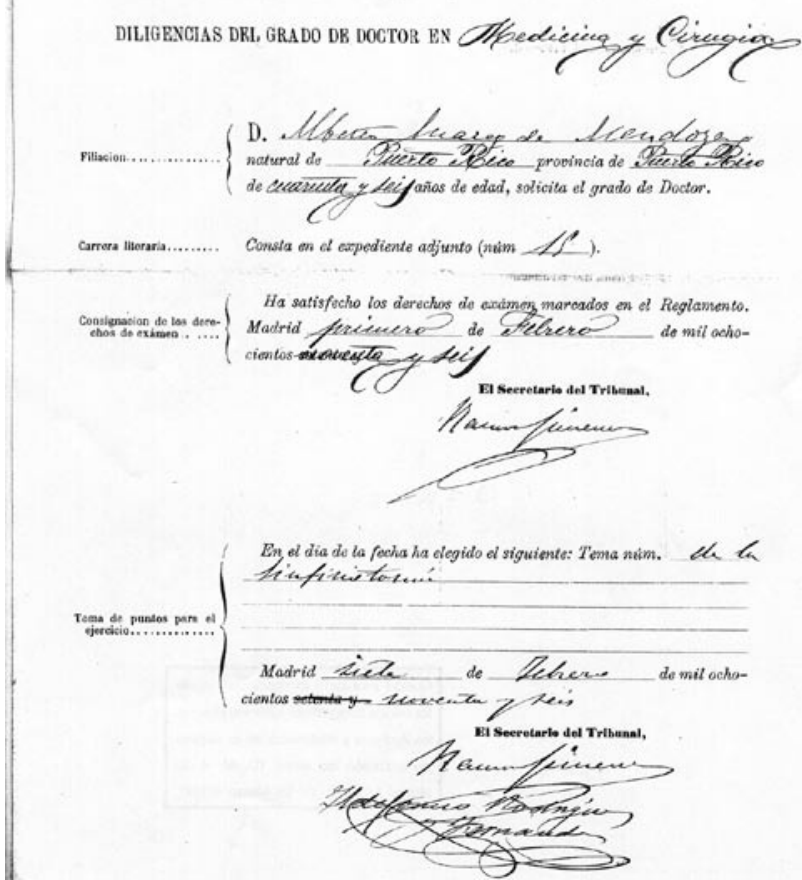

FIGURA 1. Expediente académico para la obtención del grado de doctor en la Universidad Central.

\section{TESIS DE DOCTORADO}

\section{LA SINFISEOTOMIA}

D. ALBERTO SUAREZ DE MENDOZA

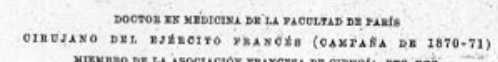

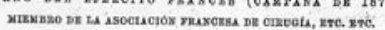

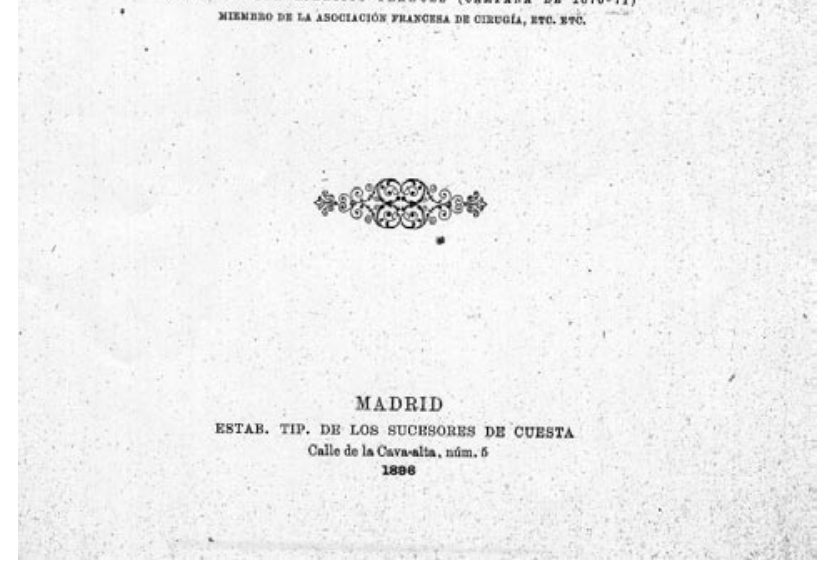

FIGURA 2. Sinfiseotomía. Tesis doctoral, Madrid, 1896. 


\section{REVISTA \\ Dx \\ MEDICINA Y CIRUGIA PRÁCTICAS

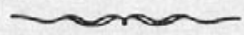 \\ LA TERAPÉtica qUIRÚRgICA Á FINES DEL SIGLO XIX \\ D. ALBERTO SUÁREZ DE MENDOZA \\ Protesor libre do enfermedades de las vias arinarias en la Foeultad do Medictos do Madrid.}

FIGURA 3. La terapéutica quirúrgica a fines del siglo XIX.

figura como profesor de Vías Urinarias; en 1897 comenzó a dar clases como "profesor auxiliar libre de Urología" en la Universidad madrileña; el Real Decreto de 12 de febrero de 1902 dio carácter oficial a la asignatura y el de la enseñanza universitaria en el que se reconocía la creación de especialidades

\section{CONGRESO HISPANO-PORTUGUESS}

DE

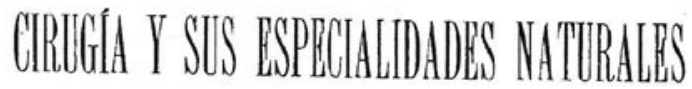

PRIMERA SESION. - MADRID: ABRIL, 1898.
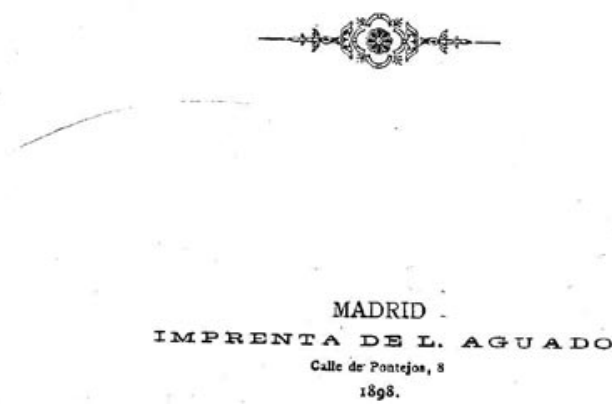

FIGURA 4. Congreso Hispano Portugués de Cirugía y sus Especialidades Naturales, Madrid, 1903. quirúrgicas, de 25 de septiembre y su ampliación de 18 de noviembre, se autorizaba a que los enseñantes libres pasaran a ser identificados como docentes universitarios de la materia impartida, con lo que automáticamente se admite al Dr. Suárez de Mendoza como profesor de la asignatura de enfermedades de la Vías Urinarias en la Facultad de Medicina de Madrid, por lo que se convierte en el primero que impartió oficialmente la disciplina urológica en la Universidad española.

Establece, a su llegada a la capital, consulta de enfermedades genitourinarias y trabaja en el Hospital de la Princesa con el Dr. Barragán hasta 1904, en que fue nombrado cirujano de la Casa de Salud y Convalecientes Pobres de Nuestra Sra. del Rosario, donde se atendían enfermedades del aparato urinario, allí mantuvo una intensa actividad quirúrgica, en 1908, afirma haber realizado 22 cistotomías suprapúbicas por litiasis, otras siete por otras patologías $y$ en cinco casos por cuadro de cistitis dolorosa; en cuatro pacientes efectuó una litotricia y en seis una uretrotomía interna; de cirugía renal practicó una nefrectomía, cinco nefropexias, dos nefrotomías por pionefrosis, una litiásica y tuberculosa la otra, además de las intervenciones de cirugía menor como circuncisiones, meatotomías, extirpaciones de quistes, etc., junto con 157 dilataciones uretrales, con un cómputo total de 144 operaciones urológicas y una mortalidad operatoria de sólo tres casos, aparte de las operaciones de cirugía general.

Inicia en 1897 la publicación de una serie de artículos urológicos principalmente en la Revista de Medicina y Cirugía Prácticas; en el titulado «La terapéutica quirúrgica a finales del siglo XIX», (Figura 3) recoge los adelantos médicos en el campo de la cirugía y su repercusión en el contexto de la sanidad y de la sociedad, así como expone los cambios en la anestesia y en las técnicas quirúrgicas. Fundó una revista bilingüe, español-francés, no urológica, editada en París y Madrid, entre 1901-1902, de título Archivos de Medicina y Cirugía Especiales, que según Maganto Pavón, tuvo continuidad hasta 1908 y en la que publicó gran cantidad de artículos suyos.

En el Congreso Hispano Portugués de Cirugía y sus Especialidades Naturales, celebrado en Madrid en 1898 (Figura 4), presentó su método de aplicar la anestesia clorofórmica (Figura 5), que había utilizado con éxito desde 1874, en doscientos pacientes sin haber tenido accidente alguno de importancia, mediante la adición de oxígeno por medio de un aparato diseñado por él para su administración (Figura 6) y cuyas principales ventajas eran poder suministrar el anestésico en dosis pequeñas y mejorar simultáneamente la oxigenación de la sangre; lo 


\section{LA MEZCLA DB OXIGBMO Y CLOROPORMO PARA LA ANESTESIA}

pon EL poctor

p. Alberto Suarez de Mendoza.

Hace cincuenta años que Morton y Jackson, utilizando durante las operaciones quirárgicas el poder anestésico del éter, que la casualidad hizo conocer $\{$ uno de ellos, realizaron uno de los descubrimientos mís importantes para la Humanidad y la Cirugfa. En el período de medio siglo, transcurrido desde entonces, se han multiplicado los trabajos sobre la anestesia, en número suficiente para formar una considerable biblioteca.

Los químicos han descubierto nuevos anestésicos; los fisiólogos han encontrado propiedades anestésicas en substancias de antiguo conocidas, y estudiado el modo de acción de todos estos agentes; los cirujanos han pedido $\leq$ la clínica las reglas a ân empíricas del empleo de estas diferentes dican a mezclíndolas de mil maras Sin el mil maneras empleados tesia, sigue siendo una verdad la antigua afirmaeión del profesor Verneuil:

\section{FIGURA 5. La mezcla de oxígeno y cloroformo para la} anestesia.

llamó «el método español», como su contribución a la ciencia española. Los cirujanos no le prestaron la debida atención a su procedimiento y cuatro años después tuvo que defender su prioridad al tener noticia de la comunicación presentada ante la Sociedad de Medicina Interna de Berlín por el profesor Heinz Wohlgemuth del buen resultado que le había proporcionado la combinación de cloroformo y oxígeno en 140 casos. Lamentablemente la aplicación del sistema del alemán tuvo aceptación en España y relegó al olvido la primacía del proyectado por el profesor Suárez de Mendoza.

Fue secretario de la sección Oficial de Urología en el XIV Congreso Internacional de Medicina celebrado en Madrid, en abril de 1903, al que aportó varias comunicaciones de cirugía general, ginecolo-

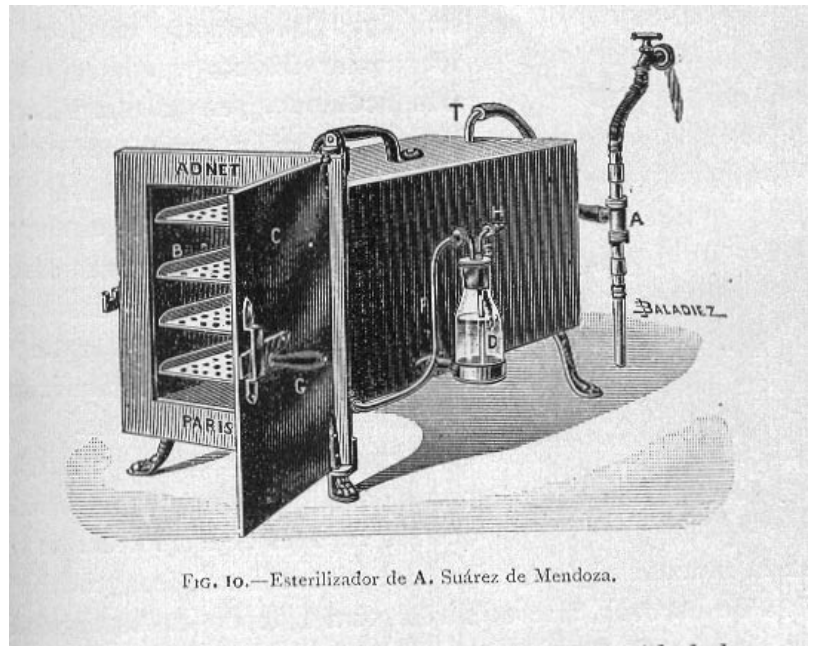

FIGURA 7. Esterilizador uretral por formaldehído, pág. 89 de su tratado.

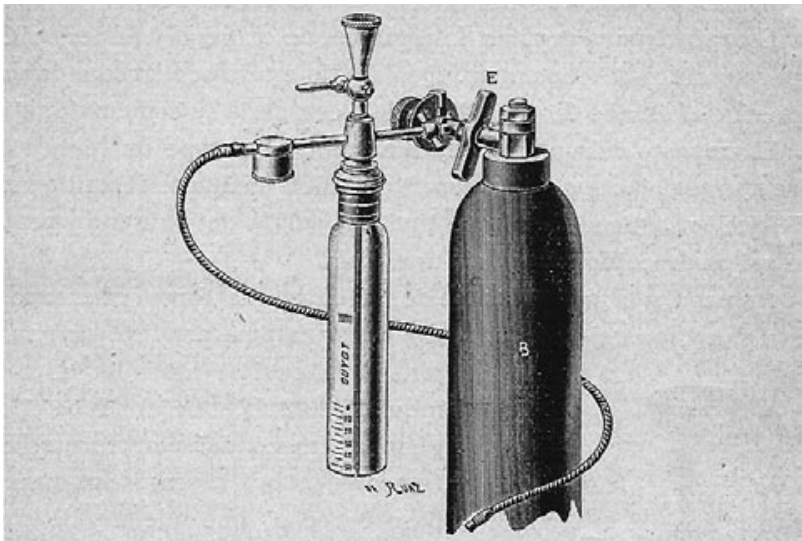

FIGURA 6. Inhalador de cloroformo y oxígeno para la anestesia, pág. 239 de su tratado.

gía y urológicas entre ellas: «Método rápido y simple para esterilizar todos los instrumentos de cirugía a la temperatura ambiente», en que presentó su invento de un «Esterilizador de instrumental por formaldehído (Figura 7), «Estado de los riñones en la anuria calculosa» y «Blenorragia desde el punto de vista médico social».

Su obra más importante es el «Diagnóstico y Tratamiento de las Enfermedades de las Vías Urinarias. Lecciones elementales», (Figura 8) publicada en Madrid en 1908, en homenaje a Félix Guyon, venerable fundador de la escuela del Necker en París, dedica estas lecciones eco lejano de sus enseñanza, en la que reúne y amplia el contenido de sus clases recogidas taquigráficamente por sus colaboradores Drs. Aguirre Gutiérrez y Pérez Grande.

La revisión de su tratado, en el que etimológicamente denomina "urología" al estudio de las orinas y "urodología" al de las enfermedades de las vías urinarias, contiene una magnífica exposición de la asignatura, con cuidadosa descripción de la parte clínica centrada en su propia experiencia y apoyada con numerosas citas de autores consagrados; dividido en cinco partes, condensa con extraordinario sentido práctico la materia de la especialidad, resalta la importancia de la anamnesis y su interpretación, describe con gran detalle y claridad los medios de exploración y las técnicas y uso del utillaje endoscópico y señala el de los rayos $X$, insiste en la trascendencia que tiene el utilizar una profilaxis antiséptica ante cualquier manipulación y aporta, también, invenciones suyas como su esterilizador de instrumental y su "Cánula de doble corriente para el lavado uretral» (Figura 9 y 10). En la parte quirúrgica dedica un capitulo a la preparación de la intervención y otro a la anestesia en la que aclara con precisión la 


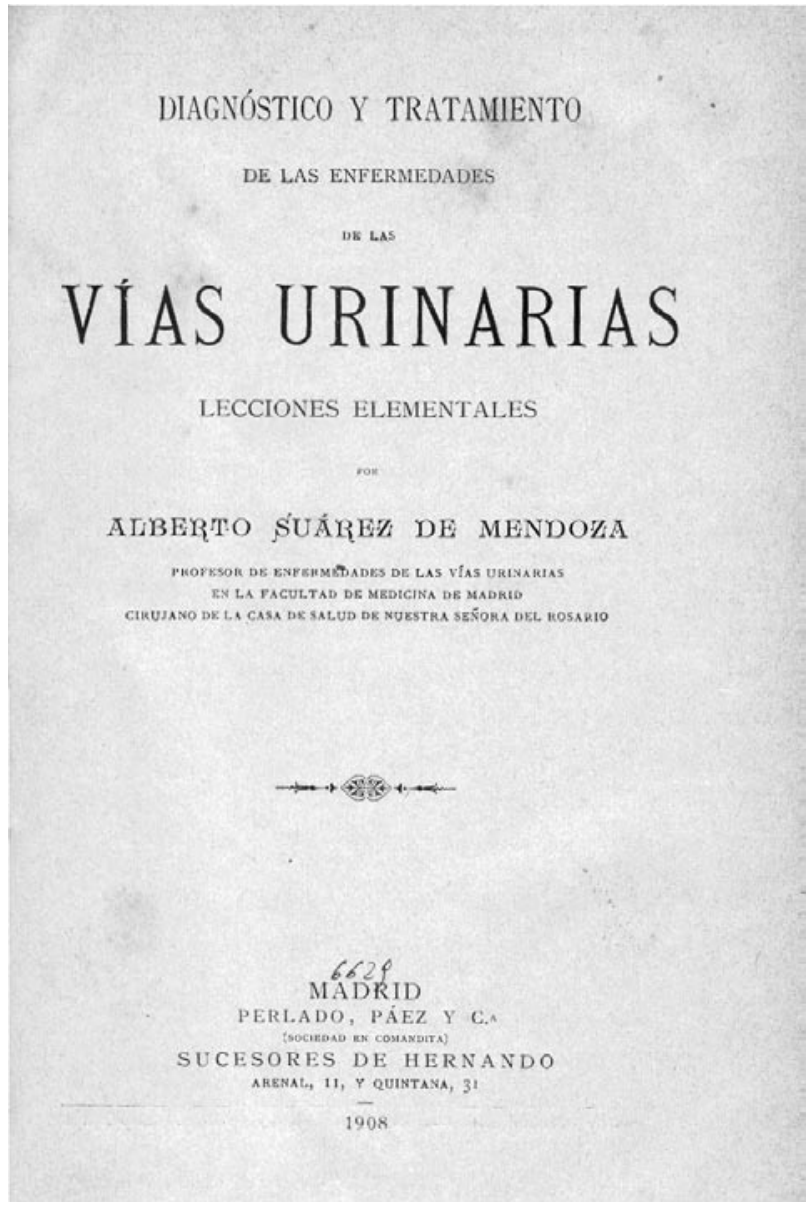

FIGURA 8. Diagnóstico y tratamiento de las enfermedades de las Vías Urinarias.

aplicación de su método, la incisión quirúrgica de la lumbotomía queda expuesta con rigor anatómico $y$, partidario de la operación de la talla suprapúbica, describe con meticulosidad la técnica y detalla la de la adenomectomía prostática de Young, por vía perineal y la hipogástrica de Freyer (Figura 11) de la que se declara partidario.

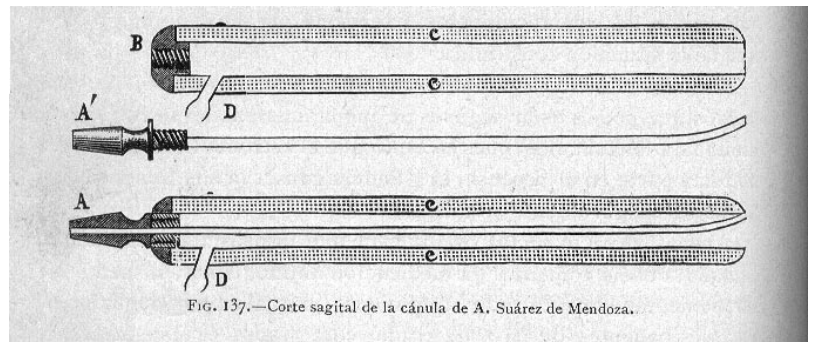

FIGURA 10. Sección de la cánula.

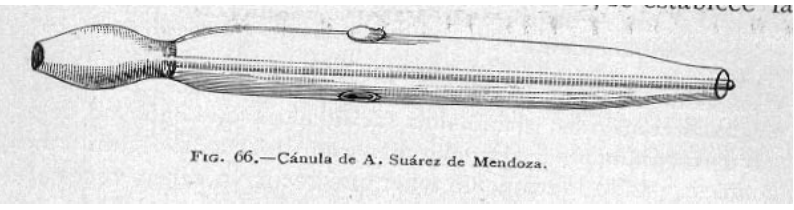

FIGURA 9. Cánula de doble corriente para el lavado uretral, pág. 275 de su tratado.

Perteneció a la Asociación Francesa de Urología, fue delegado español en el XII Congreso Francés de Cirugía de 1898, miembro del Congreso de la Prensa y de la Asociación Española de Prensa.

La última referencia suya en España es de 1910, posteriormente, una vez retirado del ejercicio profesional, se trasladó a la Costa Azul francesa, a Niza, población en la que vivió muy posiblemente el resto de sus días, sin que se conozca la fecha de su fallecimiento.

\section{PUBLICACIONES}

La sinfiseotomía, tesis doctoral, tipo. Sucesores de Cuesta, Madrid, 1896.

«La litiasis vesical en los niños», Rev. Medic. Cirug. Práct., XXI, 1897.

"Cálculos incrustados o enquistados», Rev. Medic. Cirug. Práct., XXI (537): 81-87, 1897.

«La blenorragia: diagnóstico pronóstico y tratamiento», Rev. Medic. Cirug. Práct., XXII, 1898.

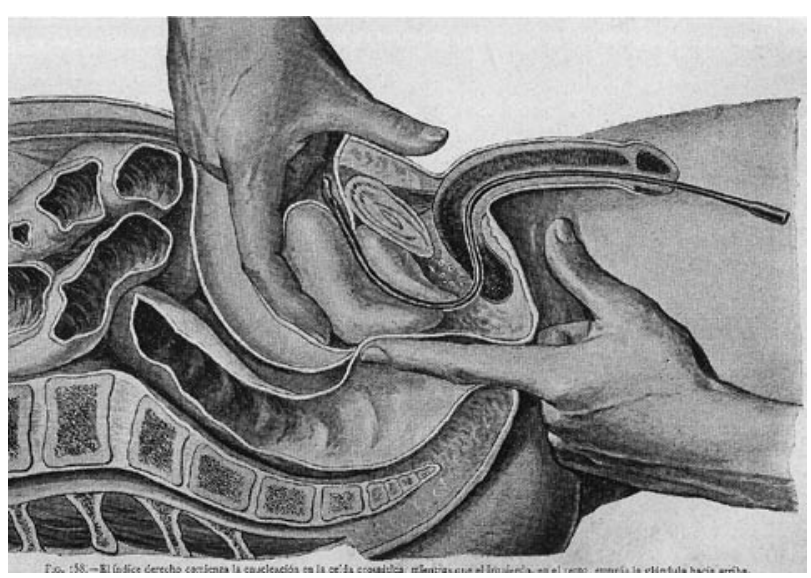

FIGURA 11. Adenomectomía prostática, técnica de Freyer, pág. 482 de su tratado. 
«La mezcla de oxigeno y cloroformo para la anestesia», Rev. Medic. Cirug. Práct., XXII, 565, 1898.

«Nuevo método de talla suprapubiana», Rev. Medic. Cirug. Práct., XXIII, (606): 695-696, 1899.

«Lecciones preparatorias al estudio clínico de las enfermedades de las vías Urinarias», Rev. Medic. Cirug. Práct. XLIV, 1899.

Cistoscopias y cateterismo de los uréteres, 49 pág., impr. Nicolás Moya, Madrid, 1899.

La terapéutica quirúrgica a finales del siglo XIX, 45 pág., impr. Nicolás Moya, Madrid, 1901.

La anuria calculosa, impr. Nicolás Moya, Madrid, 1903.

«La blenorragia desde el punto de vista médico social», Informe presentado a la sección de Dermatología y Sifilografía de la Real Academia de Medicina. Impr. Nicolás Moya, Madrid, 1903.

Diagnóstico y Tratamiento de las Enfermedades de las Vías Urinarias. Lecciones elementales, 790 pág., Idamor Moreno, Madrid, 1908.

\section{BIBLIOGRAFÍA y LECTURAS RECOMENDADAS ( lectura fundamental)}

**1. FRANCO GRANDE, A.; ÁLVAREZ ESCUDERO, J.; CORTÉS LIAÑO, J.: "Historia de la anestesia en España". Edit. Arán, Madrid, 2005.

2. MAGANTO PAVÓN, E.: "La Urología Española durante el siglo XIX y principios del XX, en Historia Biográfica y Bibliográfica de la Urología Española”. pág. 225 y 244, Edicomplet, Madrid, 2000.

*3. PÉREZ ALBACETE, M.: "100 Figuras de la Urología Española”. pág. 84-85, Tip., San Francisco, Murcia, 2005. 